\title{
Repercussões respiratórias do pneumoperitônio induzido em suínos ${ }^{1}$
}

\author{
Respiratory repercussion of pneumoperitoneum induced in pigs
}

\author{
Valéria Marques Ferreira Normando², Marcus Vinicius Henriques Brito ${ }^{3}$, Francisco Alves de Araújo Júnior ${ }^{4}$, Breno Costa \\ Maciel Albuquerque 5 \\ 1. Resumo da Dissertação apresentada á Universidade do Estado do Pará (UEPA) para a obtenção do título de Mestre em Motricidade Humana. \\ Trabalho realizado no Laboratório de Cirurgia Experimental da UEPA (LCE - UEPA) - Belém - PA \\ 2. Mestranda em Motricidade Humana da UEPA e Professora Assistente I de Fisioterapia nas enfermidades e distúrbios funcionais do sistema \\ cardiovascular e respiratório pela Universidade da Amazônia (UNAMA). \\ 3. Professor Adjunto Doutor e Coordenador da Disciplina de Técnica Operatória, Cirurgia Experimental e Anestesiologia da UEPA e Coordenador \\ do LCE - UEPA. Orientador da Dissertação. \\ 4. Graduando do Curso de Medicina da UEPA e Estagiário do LCE - UEPA. \\ 5. Graduando do Curso de Medicina da UFPA e Estagiário do LCE - UEPAUEPA.
}

\section{RESUMO}

Objetivo: Estudar, na faixa tida como segura, as repercussões respiratórias de diferentes pressões de pneumoperitônio, com dióxido de carbono, induzido em suínos. Métodos: Foram utilizados 14 suínos machos, da raça Landrace, pesando entre cinco e sete quilos, com 30 a 45 dias de idade. A amostra foi subdividida igualitariamente em Grupo A (pneumoperitônio com 10mmHg durante 60 minutos) e Grupo $B$ (pneumoperitônio com 15mmHg durante 60 minutos). Após a indução anestésica, os animais foram intubados, sendo monitorado o volume corrente (VC) e a saturação periférica de oxigênio $\left(\mathrm{SpO}_{2}\right)$. Resultados: Não houve diferença estatística nos valores do $\mathrm{VC} \mathrm{e} \mathrm{SpO}_{2}$ antes, durante e após a indução do pneumoperitônio. Conclusão: O pneumoperitônio, nos níveis pressóricos de 10 a $15 \mathrm{mmHg}$, não causou alteração significativa nos padrões respiratórios de suínos, durante o período de tempo estudado.

Descritores: Pneumoperitônio. Respiração. Complicações intraoperatórias. Laparoscopia cirúrgica. Suínos.

\begin{abstract}
Purpose: To study the respiratory repercussion of differents pneumoperitoneum pressions, with carbon dioxide, induced in pigs. Methods: It was used 14 male Landrance-race pigs, weighing between five and seven kiloragram, with 30 a 45 days of age. The sample was iqually divided in Group A (10 mmHg of intra-abdominal during 60 minuts) and Group B (15mmHg of intra-abdominal during 60 minuts). After anesthetic induction, the animals were intubed and kept under monitoring the current volume (CV) and oxigen peripheric saturation $\left(\mathrm{SpO}_{2}\right)$. Results: It not had statistical difference in $\mathrm{CV}$ and $\mathrm{SpO}_{2}$ values before, during and after the induction of pneumoperitoneum. Conclusion: Pneumoperitoneum, at the level of 10 to $15 \mathrm{mmHg}$ of intra-abdominal pression, did not cause significance alterations on pigs respiratory standart, during the time period studied.
\end{abstract}

Key words: Pneumoperitoneum. Respiration. Intraoperative Complications. Laparoscopy. Swine.

\section{Introdução}

A videocirurgia tornou-se tão popular nestes últimos 20 anos, que o domínio de sua técnica pertence a grande maioria dos cirurgiões e, sem dúvida, contribuiu soberbamente para o engrandecimento de cada especialidade que se propunha a colocá-la em prática. ${ }^{1}$

Porém, apesar do método ser minimamente invasivo, não são descartadas as possibilidades de complicações pósoperatórias, onde se destacam as complicações pulmonares, sendo consideradas fator de alta taxa de morbidade nos procedimentos cirúrgicos do abdômen superior. ${ }^{2}$

Para se dar início ao procedimento cirúrgico videolaparoscópico, deve ser realizado o pneumoperitônio, causando, conseqüentemente, aumento da pressão intra-abdominal (PIA), proporcionando deslocamento cefálico do diafragma e conseqüentemente redução dos volumes pulmonares. Portanto, esse procedimento pode levar a graves complicações cardiorrespiratórias se o volume de ar for injetado aleatoriamente ou ao gosto do cirurgião. À medida que se aumenta a PIA e melhora-se o campo, aumentam as complicações não só no trans, mas pós-operatórias também, principalmente se as operações incluírem o andar superior do abdômen. ${ }^{3}$

Iwasaka e $\mathrm{col}^{4}$, estudaram pacientes submetidos a 
colecistectomias laparoscópica e observaram diminuição da complacência pulmonar durante indução do pneumoperitônio, com posterior melhora após a desinsuflação da cavidade abdominal. Rauh e $\mathrm{col}^{1}$, avaliando o efeito do pneumoperitônio e o posicionamento do paciente na complacência pulmonar, observaram diminuição desta durante a PIA de $15 \mathrm{mmHg}$, porém sem guardar relação com o posicionamento do paciente.

Tendo em vista as possíveis deletérias alterações fisiopatológicas do pneumoperitônio, além da vasta aplicação clínica e científica da videolaparoscopia, propõe os autores avaliar as repercussões ventilatórias de diferentes pressões de pneumoperitônio induzido em suínos.

\section{Métodos}

O presente trabalho foi desenvolvido sobre aprovação do Comitê de Ética do Hospital Universitário João de Barros Barreto (HUJBB) - Belém-PA. O estudo foi conduzido obedecendo, rigorosamente, os princípios éticos do Colégio Brasileiro de Experimentação Animal (COBEA) e de acordo com a Lei Federal n ${ }^{\circ}$ 6638, de 08 de maio de 1979.

Para a realização desse estudo, foram utilizados 14 suínos da raça Landrace, machos, com idade entre 30 a 45 dias, pesando entre cinco e sete quilos, provenientes do Sistema Penal de Americano (Belém - PA). Os animais passaram por um período de adaptação de 15 dias no Laboratório de Cirurgia Experimental da Universidade do Estado do Pará, onde foi realizado o experimento, sendo mantidos em ambiente controlado, recebendo água e ração ad libitum durante todo o estudo.

A amostra foi subdividida em dois grupos, contendo sete animais cada, a saber:

Grupo $A(n=07)$ : os animais foram submetidos à insuflação contínua da cavidade abdominal com dióxido de carbono $\left(\mathrm{CO}_{2}\right)$ na pressão de $10 \mathrm{mmHg}$, por 60 minutos.

Grupo $B(n=07)$ : os animais foram submetidos à insuflação contínua da cavidade abdominal com $\mathrm{CO}_{2}$ na pressão de $15 \mathrm{mmHg}$, por 60 minutos.

Antes de serem submetidos ao procedimento cirúrgico, os animais passaram por um período de 12 horas de jejum.

A indução anestésica foi protocolada de forma que os animais pudessem permanecer sob ventilação espontânea. Para tanto, foi realizada injeção via intramuscular, na região glútea, de $2 \mathrm{ml}$ de cloridrato de tiazina associado a $2 \mathrm{ml}$ de cloridrato de ketamina. Para a manutenção anestésica, foi administrada ao animal, a cada 30 minutos, uma dose combinada de $1 \mathrm{ml}$ de cloridrato de tiazina para $1 \mathrm{ml}$ de cloridrato de ketamina, via intramuscular na região glútea.

Após dez minutos de indução anestésica, os animais foram colocados, em decúbito dorsal horizontal a zero grau, em uma mesa operatória dotada de uma calha de alumínio, forrada e revestida de espuma e campos cirúrgicos, sendo suas patas fixadas na mesa através de presilhas.

Após posicionamento adequado do animal na calha, foi iniciada a intubação oro-traqueal, auxiliada por laringoscópio com autofoco e lâmina reta de $195 \mathrm{~mm}$ de comprimento. Em seguida, instilou-se cânula traqueal $n^{\circ} 6$ e insuflou-se o "cuff" depois de verificado o posicionamento adequado da cânula, mediante da ausculta pulmonar bilateral, finalizando, a cânula

foi fixada à mandíbula do animal com fita adesiva.

Para a insuflação da cavidade abdominal, foi utilizado um cilindro de $\mathrm{CO}_{2}$ acoplado a um manômetro devidamente ajustado de forma a liberar o gás na pressão de uma atmosfera, associado a este sistema foi acoplado um fluxômetro para o controle da liberação do gás. A monitoração foi realizada por meio de manômetro de mercúrio no sistema de três vias, para a saída do gás, aferição da pressão e insuflação da cavidade abdominal pela agulha de Verres devidamente inserida na cavidade abdominal dois centímetros acima da cicatriz umbilical do animal.

Os parâmetros adotados para avaliar os padrões respiratórios foram: volume corrente (VC) e saturação periférica de oxigênio $\left(\mathrm{SpO}_{2}\right)$.

Para o acompanhamento da monitoração ventilatória foi utilizado o ventilômetro de WRIGHT modelo OHMEDA ${ }^{\circledR}$, conectado diretamente ao tubo oro-traqueal. $\mathrm{A} \mathrm{SpO}_{2}$ foi aferida utilizando-se o oxímetro de pulso modelo ACTIVE marca ECAFIX $^{\circledR}$, por meio de um sensor luminoso infravermelho adaptado a cauda do animal.

Os parâmetros acima citados foram avaliados nos seguintes tempos:

a) Pré-insuflação (Pré Ins)

b) Cinco minutos após insuflação (5 PI)

c) Trinta minutos após insuflação (30 PI)

d) Sessenta minutos após insuflação (60 PI)

e) Após cinco minutos da desinsuflação parcial da cavidade abdominal (Desins)

Os dados foram anotados no protocolo do trabalho e inseridos em banco de dados do software Epilnfo versão 6.04.

A análise estatística se deu em duas etapas, sendo na primeira etapa analisada a diferença dentro de cada grupo, verificando a significância estatística em cada fase do procedimento cirúrgico, já na segunda etapa, foi analisada a diferença das fases entre os dois grupos estudados. Para tanto, foi aplicado o Teste t de Student, sendo na primeira etapa para dados pareados e na segunda para amostras independentes. Foi adotado como índice de significância $\alpha=0,05$ ou 5\%. 
Resultados

TABELA 1 - Valores do volume corrente (VC), em cada fase do procedimento, dos animais do Grupo A (pneumoperitônio a 10mmHg)

\begin{tabular}{|c|c|c|c|c|c|c|c|c|c|}
\hline \multirow{2}{*}{ Tempo } & \multicolumn{9}{|c|}{ Animal } \\
\hline & 1 & 2 & 3 & 4 & 5 & 6 & 7 & Média & DP \\
\hline Pré Ins & 41,6 & 38,4 & 35,7 & 24 & 40 & 38,8 & 37,9 & 36,6 & 5,9 \\
\hline 5 PI & 66,6 & 35 & 27,2 & 32,6 & 35,4 & 33,3 & 49,2 & 39,9 & 13,6 \\
\hline $30 \mathrm{PI}$ & 53,1 & 47,6 & 40 & 31,5 & 19,2 & 49,1 & 50 & 41,5 & 12,3 \\
\hline $60 \mathrm{PI}$ & 48,9 & 57,6 & 66,6 & 25 & 20,8 & 46,1 & 46,5 & 44,5 & 16,5 \\
\hline Desins & 60 & 41,6 & 34,4 & 31,2 & 23 & 54,2 & 49 & 41,9 & 13,3 \\
\hline
\end{tabular}

FONTE: Protocolo do trabalho

$\mathrm{p}>0,05$ (Teste t de Student - dados pareados)

TABELA 2 - Valores do volume corrente (VC), em cada fase do procedimento, dos animais do Grupo B (pneumoperitônio a 15mmHg)

\begin{tabular}{cccccccccc}
\hline \multirow{2}{*}{ Tempo } & \multicolumn{10}{c}{ Animal } \\
\cline { 2 - 10 } & $\mathbf{1}$ & $\mathbf{2}$ & $\mathbf{3}$ & $\mathbf{4}$ & $\mathbf{5}$ & $\mathbf{6}$ & $\mathbf{7}$ & Média & DP \\
\hline Pré Ins & 34,4 & 35,7 & 24,3 & 20 & 38,4 & 72,9 & 59,3 & $\mathbf{4 0 , 7 *}$ & $\mathbf{1 8 , 9}$ \\
$\mathbf{5}$ PI & 42,8 & 47,1 & 47,3 & 40,5 & 44,4 & 61,5 & 56,4 & $\mathbf{4 8 , 6 * *}$ & $\mathbf{7 , 6}$ \\
$\mathbf{3 0}$ PI & 40,5 & 46,1 & 33,8 & 29,4 & 30,3 & 67,5 & 50 & $\mathbf{4 2 , 5 *}$ & $\mathbf{1 3 , 5}$ \\
$\mathbf{6 0}$ PI & 38,4 & 52,5 & 25,4 & 30 & 27,7 & 75,7 & 45,4 & $\mathbf{4 2 , 2}$ & $\mathbf{1 7 , 8}$ \\
Desins & 42,8 & 46 & 39,4 & 33,3 & 43,4 & 68,1 & 61,6 & $\mathbf{4 7 , 8}$ & $\mathbf{1 2 , 5}$ \\
\hline
\end{tabular}

FONTE: Protocolo do trabalho

$* \mathrm{p}<0,05$ quando comparado com o tempo Desins (Teste t de Student-dados pareados)

** $\mathrm{p}<0,05$ quando comparado com o tempo 30 PI (Teste t de Student-dados pareados)

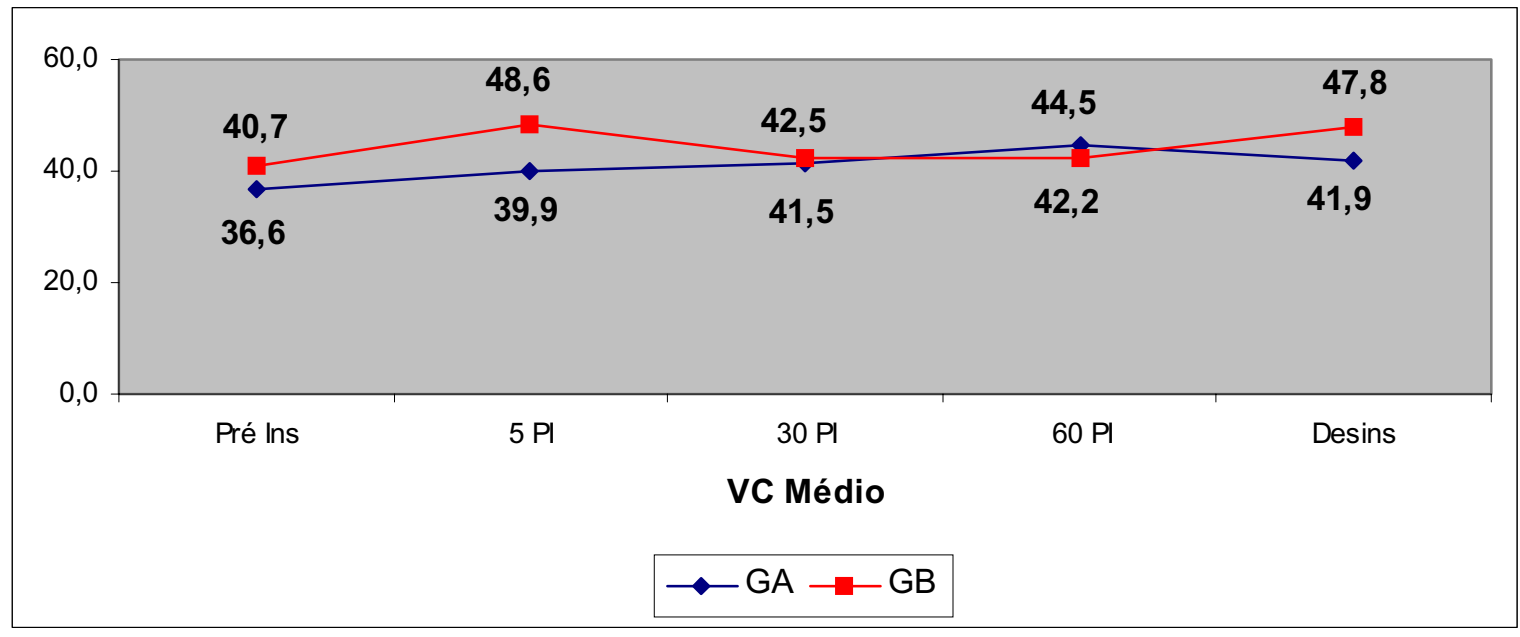

FIGURA 1 - Média dos valores do volume corrente (VC), em cada fase do procedimento, dos animais do Grupo A (pneumoperitônio a 10mmHg) e Grupo B (pneumoperitônio a 15mmHg). Fonte: protocolo do trabalho.

$\mathrm{p}>0,05$ (Teste t de Student - amostras independentes) 
TABELA 3 - Valores da saturação periférica de oxigênio $\left(\mathrm{SpO}_{2}\right)$, em cada fase do procedimento, dos animais do Grupo A (pneumoperitônio a $10 \mathrm{mmHg}$ )

\begin{tabular}{|c|c|c|c|c|c|c|c|c|c|}
\hline \multirow{2}{*}{ Tempo } & \multicolumn{9}{|c|}{ Animal } \\
\hline & 1 & 2 & 3 & 4 & 5 & 6 & 7 & GA & DP \\
\hline Pré Ins & 80 & 97 & 100 & 100 & 99 & 99 & 99 & 96,3 & 7,3 \\
\hline 5 PI & 42 & 84 & 100 & 100 & 100 & 99 & 100 & 89,3 & 21,7 \\
\hline $30 \mathrm{PI}$ & 21 & 90 & 97 & 98 & 100 & 100 & 100 & 86,6 & 29,1 \\
\hline 60 PI & 76 & 100 & 98 & 98 & 10 & 100 & 99 & 83,0 & 33,3 \\
\hline Desins & 69 & 100 & 99 & 100 & 89 & 100 & 99 & 93,7 & 11,6 \\
\hline
\end{tabular}

FONTE: Protocolo do trabalho

$\mathrm{p}>0,05$ (Teste t de Student - dados pareados)

TABELA 4 - Valores da saturação periférica de oxigênio $\left(\mathrm{SpO}_{2}\right)$, em cada fase do procedimento, dos animais do Grupo B (pneumoperitônio a $15 \mathrm{mmHg})$

\begin{tabular}{cccccccccc}
\hline \multirow{2}{*}{ Tempo } & \multicolumn{10}{c}{ Animal } \\
\cline { 2 - 10 } & $\mathbf{1}$ & $\mathbf{2}$ & $\mathbf{3}$ & $\mathbf{4}$ & $\mathbf{5}$ & $\mathbf{6}$ & $\mathbf{7}$ & $\mathbf{G B}$ & DP \\
\hline Pré Ins & 82 & 98 & 78 & 100 & 92 & 98 & 96 & $\mathbf{9 2}$ & $\mathbf{8 , 6}$ \\
$\mathbf{5}$ PI & 69 & 100 & 91 & 84 & 98 & 100 & 70 & $\mathbf{8 7 , 4 *}$ & $\mathbf{1 3 , 5}$ \\
$\mathbf{3 0}$ PI & 90 & 99 & 90 & 99 & 98 & 100 & 86 & $\mathbf{9 4 , 6}$ & $\mathbf{5 , 7}$ \\
$\mathbf{6 0}$ PI & 97 & 100 & 81 & 98 & 79 & 100 & 81 & $\mathbf{9 0 , 9}$ & $\mathbf{9 , 9}$ \\
Desins & 81 & 91 & 95 & 98 & 99 & 100 & 62 & $\mathbf{8 9 , 4}$ & $\mathbf{1 3 , 7}$ \\
\hline
\end{tabular}

FONTE: Protocolo do trabalho

$* \mathrm{p}<0,05$ quando comparado com o tempo 30 PI (Teste $t$ de

Student - dados pareados)

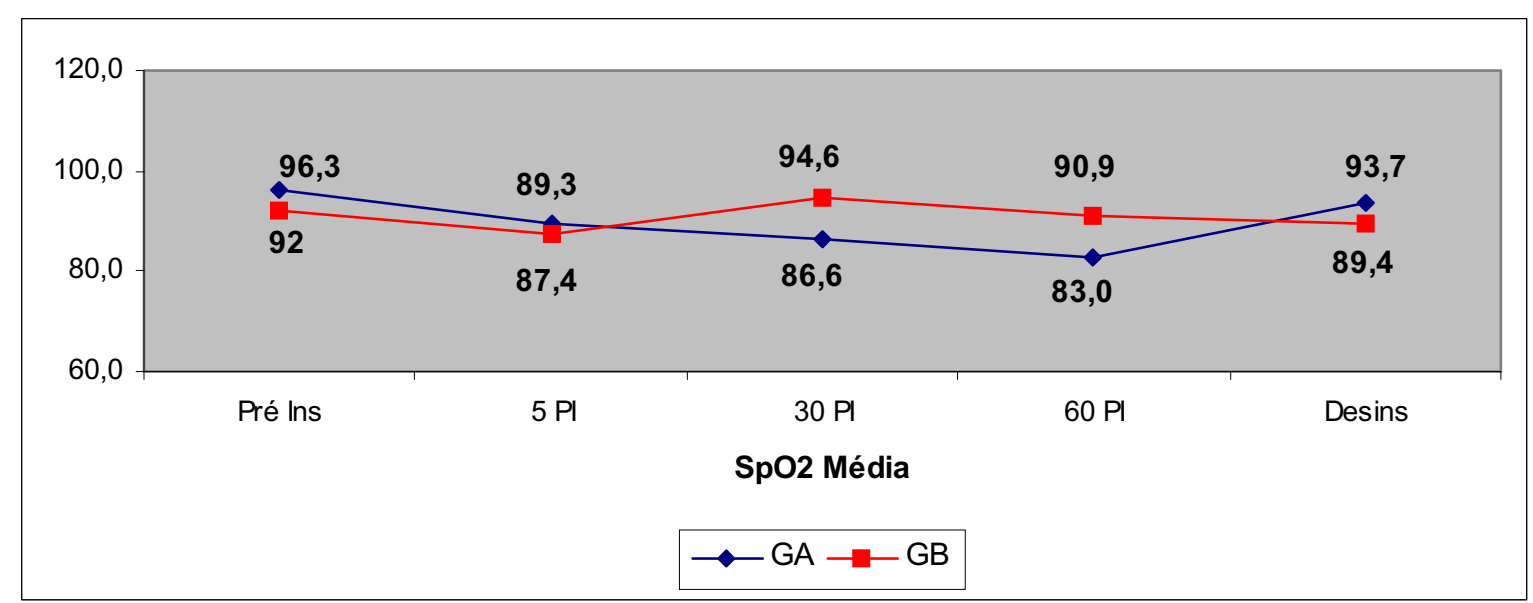

FIGURA 2 - Média dos valores da saturação periférica de $\mathrm{O} 2\left(\mathrm{SpO}_{2}\right)$, em cada fase do procedimento, dos animais do Grupo A (pneumoperitônio a 10 $\mathrm{mmHg}$ ) e Grupo B (pneumoperitônio a $15 \mathrm{mmHg}$ ). Fonte: protocolo do trabalho

$\mathrm{p}>0,05$ (Teste t de Student - amostras independentes) 


\section{Discussão}

A intervenção cirúrgica pela via laparoscópica esta contida em uma gama de operações modernas denominadas "minimamente invasivas". Estes procedimentos expadiram-se rapidamente por oferecer diversas vantagens sobre a via convencional. A diminuição da dor pós-operatória teve maior impacto humanístico, a diminuição da permanência hospitalar e o retorno precoce as atividades laborativas deram o impacto socioeconômico e as cicatrizes mínimas favoreceram os aspectos estéticos. ${ }^{5}$

Porém, esperava-se na mesma proporção, que as complicações operatórias fossem também minimizadas, o que não ocorreu, sendo demonstrados em vários estudos, fatores relevantes a nocividade do método, entretanto, com um caminho fisiopatológico diferente..$^{6,7,8,9,10,11,12}$

No presente estudo, optou-se utilizar 10 e $15 \mathrm{mmHg}$ de PIA, pois, o primeiro é considerado como valor mínimo na obtenção de um campo operatório seguro, e o segundo, por ser o valor superior utilizado na rotina dos procedimentos laparoscópicos. A indução anestésica foi protocolada de forma que os animais pudessem permanecer em ventilação espontânea, facilitando assim a monitoração ventilatória pela avaliação do VC.

Cabe ressaltar que a escolha do animal de experimentação se deu por ter suas características anatômicas de órgãos internos similares ao do ser humano, facilitando a execução do estudo proposto. Além disso, há relatos desse animal ter sido usado com êxito em experimentos anteriores..$^{13}$

Sabe-se que a insuflação da cavidade intraperitonial leva a distensão abdominal, aumento da PIA e conseqüente diminuição do movimento diafragmático, restringindo a expansibilidade pulmonar. ${ }^{14,15}$ Fato este, confirmado no estudo de Iwasaka e col ${ }^{4}$, realizado em 12 pacientes submetidos a colecistectomia laparoscópica, que observaram redução da complacência pulmonar dinâmica de 49,6 para $30,7 \mathrm{ml} / \mathrm{cmH} 20^{-1}$ durante a insuflação abdominal e, evoluindo para $45,1 \mathrm{ml} / \mathrm{cmH} 20^{-1}$ após a desinsuflação da cavidade abdominal. Rauh e $\operatorname{col}^{1}$ estudando a influência do pneumoperitônio e do posicionamento do paciente na complacência pulmonar, observaram a redução desta durante a PIA de $15 \mathrm{mmHg}$, porém o posicionamento não incrementou a alteração dos parâmetros analisados.

No presente estudo, optou-se pela análise do VC em ventilação espontânea para traduzir a quantidade de ar expirado durante a respiração normal como resposta volumétrica apresentada durante as diversas fases do experimento. ${ }^{15,16,17}$

Os resultados do VC encontrados no trabalho traduzem a idéia de independência para os valores pressóricos o que diz respeito à compressão volumétrica gerada pelo posicionamento cefálico do diafragma, pois ao se comparar os dois níveis de pressão (Figura 1), a resposta não foi significativa, independendo, portanto, dos níveis de pressão gerados entre 10 e $15 \mathrm{mmHg}$. A análise dos resultados ainda traduz a recomposição volumétrica para ambos os grupos ao final do procedimento, ou seja, após a desinsuflação (Tabelas 1 e 2).
Estes resultados são compatíveis com o estudo de Andersson e $\mathrm{col}^{18}$, que estudaram nove pacientes submetidos a colecistectomia laparoscópica, encontraram oscilação volumétrica na análise do VC durante o procedimento com estabilidade ao término.

Para a complementação da monitoração ventilatória, optou-se pela análise da $\mathrm{SpO}_{2}$, a qual oferece subsídios para a adequação da oxigenação e ventilação. ${ }^{19}$

Os resultados encontrados revelam que as respostas não foram estatisticamente significantes quando comparados os cinco tempos operatórios para dados pareados (Tabela 3 e 4) e os dois níveis de pressão de 10 e $15 \mathrm{mmHg}$ para amostras independentes (Figura 2), ilustrando a proximidade nos valores monitorados através da oximetria de pulso. A estabilidade adequou-se a ventilação espontânea sem a necessidade do enriquecimento de $\mathrm{O}_{2}$ e revelou o equilíbrio ventilatório independente do nível de pressão utilizado.

As variações nos dados monitorados não responderam de forma significativa a inadequação da relação ventilação/perfusão durante o procedimento. Estes dados são compatíveis a resposta do comportamento ventilatório monitorado pelo VC. A desigualdade na distribuição da ventilação para áreas pulmonares não-dependentes, não resultou numa relação ventilação/perfusão inadequada durante o procedimento.

$\mathrm{A} \mathrm{SpO}_{2}$ na hemoglobina foi considerada por Gandara e col ${ }^{20}$, em seu estudo como método de monitorização das alterações respiratórias durante a colecistectomia laparoscópica, utilizandose três técnicas anestésicas diferenciadas. A redução deste parâmetro revelou-se estatisticamente significativo durante o pneumoperitônio, estabilizando-se posteriormente sem apresentar diferenças entre os agentes anestésicos.

Diante do exposto, os resultados encontrados nesse estudo, a principio, não justificariam as possíveis complicações pulmonares pós-laparoscópica, uma vez que ocorreu estabilidade ventilatória após a desinsuflação da cavidade abdominal, entretanto não são descartadas a possibilidade de irritação frênica ocasiona pela localização do pneumoperitônio residual. Além disso, torna-se relevante a realização de estudos avaliando a gasometria arterial, a qual subsidiaria o referencial do equilíbrio ventilação/perfusão. Desta forma, esta linha metodológica poderá refletir em pesquisas futuras que acompanhem e enriqueçam a literatura científica.

\section{Conclusão}

O pneumoperitônio, nos níveis pressóricos de 10 a $15 \mathrm{mmHg}$, não causou alteração significativa nos padrões respiratórios de suínos, durante o período de tempo estudado.

\section{Referências}

1 - Rauh R, Hemmerling TM, Rist M, Jacob KE. Influence of pneumoperitoneum and patient positioning on respiratory system complience. J Clin Anesth. 2001; 13: 361-5.

2 - Chuter TAM, Weissman C, Mathews DM, Starker PM. Effects of incetive spirometry on diaphragmatic function in postoperative pa- 
tients. Surgery. 1989; 105: 488-98.

3 - Mergh AC, Oliveira CHS. Alterações pulmonares na cirurgia videolaparoscópica. Rev Bras Anestesiol. 1996; 46(4): 289-94.

4 - Iwasaka H, Miyakawa H, Yamamoto H, Kitano T, Taniguchi K, Honda N. Respiratory mechanics and arterial blood gases during and after laparoscopic cholecystectomy. Can J Anesth. 1996; 43(2): 129-33.

5 - Valezi AC, Rahal F. Repercussões do pneumoperitônio sobre o sistema venoso dos membros inferiores: estudo em porcas. Rev Col Bras Cir. 1999; 26(1): 45-9.

6 - Benhamou D, Simonneau G, Poynard T, Goldman M, Chaput JC, Duroux P. Diaphragm fuction is not impaired by pneumoperitoneum after laparoscopy. Arch Surg. 1993; 128: 430-2.

7 - Salvatore MM, Quinn JV, Slotman GJ. Hemodynamic effects os carbon dioxide pneumoperitoneum during mechanical ventilation and positive end-expiratory pressure. J Trauma. 1993; 35(4): 6138.

8 - Cohen E, Mier A, Heywood P, Murphy K, Boultbee J, Guz A. Excursión-volume relation of the right hemidiaphragm mesured by ultrasonography and respiratory airflow measurements. Thorax. 1994; 29: 885-9.

9 - McKenzi DK, Gandevia SC, Gorman RB, Southon FCG. Dynamic changes in the zone of apposition nd diaphragm length during maximal respiratory efforts. Thorax. 1994; 49: 634-8.

10 - Goottesman E, McCool FD. Ultrasound evaluation of the paralyzed diaphragm. Am J Resp Crit Care Med. 1997; 155: 1570-4.

11 - Hachenberg T, Ebel C, Czorny M, Thomas H, Wendt M. Intrathoracic and pulmonary blood volume during $\mathrm{CO} 2$ pneumoperitoneum in humans. Acta Anaesthesiol Scand. 1997; 42(7): 794-8.

12 - Loeckinger A, Kleinsasser A, Hoermann C, Krismer A, Gassner
M, Keller C, Puehringer F, Linder KH. Inert gas exchange during pneumoperitoneum at incremental values of positive end-expiratory pressure. Anesth Analg. 2000; 90: 466-71.

13 - Rodrigues FCM. Efeitos do pneumoperitônio com gás carbônico e hélio na pressão de perfusão cerebral m modelo experimental de trauma crânio-encefálico [Tese - Doutorado]. Faculdade de Ciências Médicas da Santa Casa de São Paulo; 1995.

14 - Bartlett RH. Clínicas cirúrgicas da América do Norte. Rio de Janeiro (RJ): Interamericana; 1980.

15 - Wittgen CM, Anrus CH, Fitzgerald SD, Baudendistel LJ, Dahms TE, Kaminski DL. Analysis of the hemodynamic and ventilatory effects of laparoscopic cholecystectomy. Arch Surg. 1991; 126(8): 997-1000.

16 - West JB. Fisiologia respiratória moderna. São Paulo (SP): Ed. Manole; 1978.

17 - Couture JG, Chartrand D, Gagner M, Bellemare F. Diaphragmatic and abdominal muscle activity after endoscopic cholecystectomy. Anesth Anal 1994; 78: 733-9.

18 - Andersson L, Lagerstrand L, Thorne A, Sollevi A, Brodin LA, Odeberg-Wenerman S. Effects of $\mathrm{CO} 2$ pneumoperitoneum on ventilation-perfusion relationships during laparoscopic cholecystectomy. Acta Anaesthesiol Scand 2002; 46: 552-60.

19 - McDermott JP, Regan MC, Page R, Stokes MA, Barry K, Moriarty DC, Caushaj PF, Fitzpatrick JM, Gorey TF. Cardiorespiratory effects of laparoscopy with and without gas insulflation. Arch Surg. 1995; 130(9): 984-8.

20 - Gándara MV, Vega DS, Escriú N, Olmedilla C, Pérez-Mencia MT, Zueras R, López A. Alteraciones respiratorias durante la colecistectomia laparoscopica. Estudio comparativo de tres tecnicas anetesicas. Rev Esp Anestesiol Reanim. 1997; 44(5): 177-81.
Correspondência:

Valéria Marques Ferreira Normando

Av. Alcindo Cacela, 727

66060-000 Belém - PA

Tel.: (91)246-1233

Fax: (91)266-3184

normando@amazon.com.br
Conflito de interesse: nenhum

Fonte de financiamento: nenhuma
Recebimento: 23/08/2004

Revisão: 02/09/2004

Aprovação: 19/10/2004

\section{Como citar este artigo:}

Normando VMF, Brito MVH, Araújo Jr FA, Albuquerque BCM. Repercussões respiratórias do pneumoperitônio induzido em suínos. Acta Cir Bras. [serial online] 2004 Nov-Dez;19(6). Disponível em URL: http://www.scielo.br/acb [também em CDROM].

*Figuras coloridas disponíveis em www.scielo.br/acb 